\title{
Spectral stability of Schrödinger operators with subordinated complex potentials
}

\author{
Luca Fanelli, ${ }^{a}$ David Krejčiř́rik ${ }^{b}$ and Luis Vega ${ }^{c}$
}

a) Dipartimento di Matematica, SAPIENZA Università di Roma, P. le Aldo Moro 5, 00185 Roma; fanelli@mat.uniroma1.it.

b) Department of Mathematics, Faculty of Nuclear Sciences and Physical Engineering, Czech Technical University in Prague, Trojanova 13, 12000 Prague 2, Czechia; david.krejcirik@fjfi.cvut.cz.

c) Departamento de Matemáticas, Universidad del Pais Vasco, Aptdo. 644, 48080 Bilbao, \& Basque Center for Applied Mathematics (BCAM), Alameda Mazarredo 14, 48009 Bilbao, Spain; luis.vega@ehu.es83lvega@bcamath.org.

6 December 2016

\begin{abstract}
We prove that the spectrum of Schrödinger operators in three dimensions is purely continuous and coincides with the non-negative semiaxis for all potentials satisfying a form-subordinate smallness condition. By developing the method of multipliers, we also establish the absence of point spectrum for Schrödinger operators in all dimensions under various alternative hypotheses, still allowing complex-valued potentials with critical singularities.
\end{abstract}

\section{Introduction}

Let $H_{0}$ be the free Hamiltonian, i.e. the self-adjoint operator in $L^{2}\left(\mathbb{R}^{d}\right)$ associated with the quadratic form

$$
h_{0}[\psi]:=\int_{\mathbb{R}^{d}}|\nabla \psi|^{2}, \quad \mathrm{D}\left(h_{0}\right):=H^{1}\left(\mathbb{R}^{d}\right) .
$$

Let $V: \mathbb{R}^{d} \rightarrow \mathbb{C}$ be a measurable function which is form-subordinated to $H_{0}$ with the subordination bound less than one, i.e.,

$$
\exists a<1, \quad \forall \psi \in H^{1}\left(\mathbb{R}^{d}\right), \quad \int_{\mathbb{R}^{d}}|V||\psi|^{2} \leq a \int_{\mathbb{R}^{d}}|\nabla \psi|^{2}
$$

In view of the criticality of $H_{0}$ in low dimensions, (1) is admissible for $d \geq 3$ only, to which we restrict in the sequel.

Assumption (11) in particular means that the quadratic form

$$
v[\psi]:=\int_{\mathbb{R}^{d}} V|\psi|^{2}, \quad \mathrm{D}(v):=\left\{\psi \in L^{2}\left(\mathbb{R}^{d}\right): \int_{\mathbb{R}^{d}}|V \| \psi|^{2}<\infty\right\} .
$$

is relatively bounded with respect to $h_{0}$ with the relative bound less than one. Consequently, the sum $h_{V}:=h_{0}+v$ is a closed form with $\mathrm{D}\left(h_{V}\right)=H^{1}\left(\mathbb{R}^{d}\right)$ which gives rise to an m-sectorial operator $H_{V}$ in $L^{2}\left(\mathbb{R}^{d}\right)$ via the representation theorem ( $c f$. [16, Thm. VI.2.1]). It is customary to write

$$
H_{V}=H_{0} \dot{+} V,
$$

but we stress that this generalised sum in the sense of forms differs from the ordinary operator sum.

The purpose of this paper is to show that condition (11) is sufficient to guarantee that the spectra of $H_{0}$ and $H_{V}$ coincide, at least under some extra hypotheses. 
Recall that the spectrum, $\sigma(H)$, of a closed operator $H$ in a complex Hilbert space $\mathcal{H}$ is determined by the set of points $\lambda \in \mathbb{C}$ for which $H-\lambda: \mathrm{D}(H) \rightarrow \mathcal{H}$ is not bijective. Three disjoint subsets of $\sigma(H)$ that exhaust the spectrum are distinguished: the point spectrum $\sigma_{\mathrm{p}}(H):=\{\lambda \in \mathbb{C}: H-\lambda$ is not injective $\}$, the continuous spectrum $\sigma_{\mathrm{c}}(H):=\left\{\lambda \in \sigma(H) \backslash \sigma_{\mathrm{p}}(H): \overline{\mathrm{R}(H-\lambda)}=\mathcal{H}\right\}$ and the residual spectrum $\sigma_{\mathrm{r}}(H):=\left\{\lambda \in \sigma(H) \backslash \sigma_{\mathrm{p}}(H): \overline{\mathrm{R}(H-\lambda)} \neq \mathcal{H}\right\}$.

The spectrum of $H_{0}$ is well known to be purely continuous, in fact $\sigma\left(H_{0}\right)=\sigma_{\mathrm{c}}\left(H_{0}\right)=[0,+\infty)$. In this paper we show that this spectral property is preserved by condition (11) provided that $d=3$.

Theorem 1. Let $d=3$ and assume (1). Then $\sigma\left(H_{V}\right)=\sigma_{\mathrm{c}}\left(H_{V}\right)=[0,+\infty)$.

The theorem is proved in four steps:

(i) Absence of the residual spectrum;

Section 1

(ii) Absence of the point spectrum;

Section 2

(iii) Absence of the continuous spectrum in $\mathbb{C} \backslash[0,+\infty)$;

Section 3 .

(iv) Inclusion of $[0,+\infty)$ in the spectrum;

Section 4

Property (i) follows at once (in any dimension): Since the adjoint operator satisfies $H_{V}^{*}=H_{\bar{V}}=\mathcal{T} H_{V} \mathcal{T}$, where $\mathcal{T}$ is the complex-conjugation operator defined by $\mathcal{T} \psi:=\bar{\psi}, H_{V}$ is $\mathcal{T}$-self-adjoint (cf. [ [8, Sec. III.5]) and as such it has no residual spectrum (cf. [3]). The absence of eigenvalues (ii) is established in Section 2 by means of an argument reminiscent of the Birman-Schwinger principle, but we emphasise that positive eigenvalues are excluded as well. Property (iii) is proved by a modified version of the previous argument in Section 3. Finally, in Section 4 we establish (iv) with help of an abstract quadratic-form criterion for the inclusion of points in the spectrum.

The present paper is primarily motivated by a recent interest in spectral theory of Schrödinger operators with complex potential, see [1, 12, 4, 19, 6, 21, 11, 7, 9, 13. However, the role of hypothesis (1) to have the conclusion of Theorem 1 seems to be new in the self-adjoint case, too.

As a matter of fact, Simon established the absence of eigenvalues in the self-adjoint case for $d=3$ already in [22, Thm. III.12] (see also [20, Thm. XIII.21]) by assuming

$$
\|V\|_{R}^{2}:=\iint_{\mathbb{R}^{3} \times \mathbb{R}^{3}} \frac{|V(x)||V(y)|}{|x-y|^{2}} d x d y<(4 \pi)^{2} .
$$

The extension of his method to complex potentials is straightforward. However, notice that our assumption (11) is weaker. Indeed, (1) is equivalent to (25), while

$$
\left\||V|^{1 / 2} H_{0}^{-1 / 2}\right\|^{2}=\left\||V|^{1 / 2} H_{0}^{-1}|V|^{1 / 2}\right\| \leq\left\||V|^{1 / 2} H_{0}^{-1}|V|^{1 / 2}\right\|_{\mathrm{HS}}=\frac{\|V\|_{R}}{4 \pi}
$$

where $\|\cdot\|$ and $\|\cdot\|_{\text {HS }}$ denote the operator and Hilbert-Schmidt norms in $L^{2}\left(\mathbb{R}^{3}\right)$, respectively. The last equality in (5) follows with help of the explicit formula for the Green function (23) in $\mathbb{R}^{3}$.

To be more specific, notice that, by virtue of the classical Hardy inequality

$$
\forall \psi \in H^{1}\left(\mathbb{R}^{d}\right), \quad \int_{\mathbb{R}^{d}}|\nabla \psi|^{2} \geq\left(\frac{d-2}{2}\right)^{2} \int_{\mathbb{R}^{d}} \frac{|\psi(x)|^{2}}{|x|^{2}} d x,
$$

our hypothesis (1) is in particular satisfied for potentials $V$ verifying

$$
|V(x)| \leq a\left(\frac{d-2}{2}\right)^{2} \frac{1}{|x|^{2}}
$$

for almost every $x \in \mathbb{R}^{d}$. However, the Hardy potential on the right hand side of this inequality does not even belong to the Rollnik class characterised for $d=3$ by the norm $\|\cdot\|_{R}$ in (44). Furthermore, the location of the continuous spectrum without the hypothesis that $V$ belongs to the Rollnik class (which ensures the finiteness of the Hilbert-Schmidt norm above) is less evident in our more general setting.

Our Theorem 1 is also an improvement upon the non-self-adjoint situation considered by Frank in [11, Thm. 2]. First, he establishes the absence of eigenvalues outside $[0,+\infty)$ only. Second, his assumption to get the conclusion of Theorem 1 for $d=3$ is

$$
\int_{\mathbb{R}^{3}}|V(x)|^{3 / 2} d x<\frac{3^{3 / 2}}{4 \pi^{2}}
$$


which is again stronger than ours (10. Indeed, by the Hölder and Sobolev inequalities,

$$
\int_{\mathbb{R}^{3}}|V||\psi|^{2} \leq\left(\int_{\mathbb{R}^{3}}|V|^{3 / 2}\right)^{2 / 3}\left(\int_{\mathbb{R}^{3}}|\psi|^{6}\right)^{1 / 3} \leq\left(\int_{\mathbb{R}^{3}}|V|^{3 / 2}\right)^{2 / 3} \frac{2^{4 / 3}}{3 \pi^{4 / 3}} \int_{\mathbb{R}^{3}}|\nabla \psi|^{2},
$$

for all $\psi \in H^{1}\left(\mathbb{R}^{3}\right)$. As an example, the Hardy potential on the right hand side of (7) makes the left hand side of (8) infinite, while it is an admissible potential for our Theorem 1] Finally, let us mention that Frank and Simon have noticed recently in [13] that even positive eigenvalues can be excluded.

Our hypothesis (1D) is of course intrinsically a smallness condition about $V$. But it is interesting to notice that it involves potentials with quite rough local singularities, e.g. (7). It seems that such potentials are not typically covered by previous works on the exclusion of embedded eigenvalues, even in the self-adjoint case; see [15, 17] to quote just the most recent results based on Carleman's estimates.

The extension of Theorem 11 to higher dimensions is not obvious, since our method relies on the pointwise inequality for Green's functions (27), which does not hold for $d>3$. As an alternative approach, in Section 5 , we develop the technique of multipliers for Schrödinger operators with complexvalued potentials and prove the absence of eigenvalues in any dimension under a stronger hypothesis.

Theorem 2. Let $d \geq 3$ and assume

$$
\exists b<\frac{d-2}{5 d-8}, \quad \forall \psi \in H^{1}\left(\mathbb{R}^{d}\right), \quad \int_{\mathbb{R}^{d}} r^{2}|V|^{2}|\psi|^{2} \leq b^{2} \int_{\mathbb{R}^{d}}|\nabla \psi|^{2},
$$

where $r(x):=|x|$. Then $\sigma_{\mathrm{p}}\left(H_{V}\right)=\varnothing$.

Notice that (10) follows as a consequence of (10) by means of the Schwarz inequality and the classical Hardy inequality (6). Indeed, (10) and (6) yield

$$
\int_{\mathbb{R}^{d}}|V||\psi|^{2} \leq\|r V \psi\|\left\|\frac{\psi}{r}\right\| \leq \frac{2 b}{d-2} \int_{\mathbb{R}^{d}}|\nabla \psi|^{2} .
$$

for all $\psi \in H^{1}\left(\mathbb{R}^{d}\right)$, and $b<(d-2) / 2$ due to the restriction in (10).

Both (11) and (10) are smallness assumptions about $V$. Our next step is to look for some alternative conditions which guarantee the absence of eigenvalues for $H_{V}$, in all dimensions $d \geq 3$. The idea is to modify the proof of Theorem 2 by splitting the real and imaginary parts of the potential $V$ and treating them separately. In order to include potentials which are not necessarily subordinated in the spirit of (10), we consider the space

$$
\mathcal{D}\left(\mathbb{R}^{d}\right):={\overline{C_{0}^{\infty}\left(\mathbb{R}^{d}\right)}}^{\|\cdot\| \|}, \quad\||\psi|\|^{2}:=\int_{\mathbb{R}^{d}}|\nabla \psi|^{2}+\int_{\mathbb{R}^{d}}(\Re V)_{+}|\psi|^{2}+\int_{\mathbb{R}^{d}}|\psi|^{2},
$$

where we have introduced the notation $f_{ \pm}:=\max \{ \pm f, 0\}$ for any measurable function $f: \mathbb{R}^{d} \rightarrow \mathbb{R}$. Clearly, $\mathcal{D}\left(\mathbb{R}^{d}\right)$ is continuously embedded in $H^{1}\left(\mathbb{R}^{d}\right)$ and it coincides with the latter as a set if (1) holds. The form $h_{V}^{(1)}[\psi]:=\int_{\mathbb{R}^{d}}|\nabla \psi|^{2}+\int_{\mathbb{R}^{d}}(\Re V)_{+}|\psi|^{2}, \mathrm{D}\left(h_{V}^{(1)}\right):=\mathcal{D}\left(\mathbb{R}^{d}\right)$, is closed by definition. Assuming now only that $(\Re V)_{\text {- }}$ and $\Im V$ are form-subordinated to $H_{0}$ with the subordination bound less than one (cf. (14) and (16) below), the sum $h_{V}:=h_{V}^{(1)}+h_{V}^{(2)}$ with $h_{V}^{(2)}[\psi]:=-\int_{\mathbb{R}^{d}}(\Re V)_{-}|\psi|^{2}+\int_{\mathbb{R}^{d}} \Im V|\psi|^{2}$ is a closed form with $\mathrm{D}\left(h_{V}\right)=\mathcal{D}\left(\mathbb{R}^{d}\right)$. Of course, $h_{V}$ coincides with the previously defined form under the hypothesis (11). In this more general setting, we also denote by $H_{V}$ the m-sectorial operator associated with $h_{V}$.

Now we are in a position to state the main result about the absence of eigenvalues for $H_{V}$ under natural conditions on $V$.

Theorem 3. Let $d \geq 3$ and assume that there exist non-negative numbers $b_{1}, b_{2}, b_{3}$ satisfying

$$
b_{1}^{2}<1-\frac{2 b_{3}}{d-2}, \quad b_{2}^{2}+2 b_{3}+\frac{1}{4} \sqrt{b_{3}}\left(\frac{2}{d-2}\right)^{\frac{3}{2}}<1,
$$


such that, for all $\psi \in \mathcal{D}\left(\mathbb{R}^{d}\right)$,

$$
\begin{array}{r}
\int_{\mathbb{R}^{d}}(\Re V)_{-}|\psi|^{2} \leq b_{1}^{2} \int_{\mathbb{R}^{d}}|\nabla \psi|^{2}, \\
\int_{\mathbb{R}^{d}}\left[\partial_{r}(r \Re V)\right]_{+}|\psi|^{2} \leq b_{2}^{2} \int_{\mathbb{R}^{d}}|\nabla \psi|^{2}, \\
\int_{\mathbb{R}^{d}} r^{2}|\Im V|^{2}|\psi|^{2} \leq b_{3}^{2} \int_{\mathbb{R}^{d}}|\nabla \psi|^{2},
\end{array}
$$

where $\partial_{r} f(x):=\frac{x}{|x|} \cdot \nabla f(x)$. Then $\sigma_{\mathrm{p}}\left(H_{V}\right)=\varnothing$.

We recall that (14) and (16) ensure that $h_{V}^{(2)}$ is subordinated to $h_{V}^{(1)}$ with the subordination bound less than one, so $H_{V}$ is indeed well defined. A brief comparison between Theorems 1 , 2 and 3 is in order:

- If $\Im V=0$, namely $V$ is real-valued, then $b_{3}$ can be chosen to be equal to zero and condition (13) then reads $b_{1}<1, b_{2}<1$. In this case, the subordination assumption (11) implies (14). However, we stress that conditions (14) and (15) are not unsigned, contrary to the case of (1). In particular, a large class of repulsive potentials such as the Coulomb-type interaction $V(x)=c|x|^{-1}$ with any $c>0$ satisfy (14) and (15), although the subordination (11) fails.

- On the other hand, if $\Re V=0$, namely $V$ is purely imaginary-valued, then (144), (15) are fulfilled and one just needs to assume (16) with

$$
\sqrt{b_{3}}<8\left[\left(\frac{2}{d-2}\right)^{\frac{3}{2}}+\sqrt{\left(\frac{2}{d-2}\right)^{3}+128}\right]^{-1} .
$$

This hypothesis is better than condition (10) of Theorem 2 and represents a completely new result, to our knowledge. However, for general complex-valued potentials $V$, the interest of Theorem 2 consists in that it requires no conditions on the derivatives of $V$.

The techniques used to prove Theorems 2 and 3 permit to handle more general lower-order perturbations of $H_{0}$. It is of particular interest for the electromagnetic Hamiltonian $H_{A, V}$ that we introduce as follows. Given a magnetic potential $A \in L_{\mathrm{loc}}^{2}\left(\mathbb{R}^{d} ; \mathbb{R}^{d}\right)$ and denoting by $\nabla_{A}:=\nabla+i A$ the magnetic gradient, we now consider the space

$$
\mathcal{D}_{A}\left(\mathbb{R}^{d}\right):=\overline{C_{0}^{\infty}\left(\mathbb{R}^{d}\right)}\|\cdot\|_{A}, \quad\left\|\left.\left|\psi \|_{A}^{2}:=\int_{\mathbb{R}^{d}}\right| \nabla_{A} \psi\right|^{2}+\int_{\mathbb{R}^{d}}(\Re V)_{+}|\psi|^{2}+\int_{\mathbb{R}^{d}}|\psi|^{2},\right.
$$

and introduce the form $h_{A, V}[\psi]:=\int_{\mathbb{R}^{d}}\left|\nabla_{A} \psi\right|^{2}+\int_{\mathbb{R}^{d}} V|\psi|^{2}, \mathrm{D}\left(h_{A, V}\right):=\mathcal{D}_{A}\left(\mathbb{R}^{d}\right)$. If $V$ is such that (19) and (21) below hold, then $h_{A, V}$ is closed. We denote by $H_{A, V}$ the m-sectorial operator associated with $h_{A, V}$. We next denote by $B:=\nabla A-(\nabla A)^{t} \in \mathcal{M}_{d \times d}(\mathbb{R})$ the magnetic field generated by $A$. (For $d=3, B$ may be identified with $\operatorname{curl} A$, in the sense that $B v=\operatorname{curl} A \times v$ for all $v \in \mathbb{R}^{3}$, where the cross denotes the vectorial product.) Following a notation introduced in [10, we also define

$$
B_{\tau}(x):=\frac{x}{|x|} \cdot B(x) .
$$

(A non-trivial example of magnetic field with $B_{\tau}=0$ is given in dimension $d=3$ by the magnetic potential $A(x)=|x|^{-2}\left(-x_{2}, x_{1}, 0\right)$.)

The last result of this manuscript is an analogue of Theorem 3 in the presence of an external magnetic field.

Theorem 4. Let $d \geq 3, A \in L_{\mathrm{loc}}^{2}\left(\mathbb{R}^{d} ; \mathbb{R}^{d}\right)$ and assume that there exist non-negative numbers $b_{1}, b_{2}, b_{3}$ satisfying (13) such that, for all $\psi \in \mathcal{D}_{A}\left(\mathbb{R}^{d}\right)$,

$$
\begin{aligned}
\int_{\mathbb{R}^{d}}(\Re V)_{-}|\psi|^{2} & \leq b_{1}^{2} \int_{\mathbb{R}^{d}}\left|\nabla_{A} \psi\right|^{2}, \\
\int_{\mathbb{R}^{d}}\left[\partial_{r}(r \Re V)\right]_{+}|\psi|^{2} & \leq b_{2}^{2} \int_{\mathbb{R}^{d}}\left|\nabla_{A} \psi\right|^{2}, \\
\int_{\mathbb{R}^{d}} r^{2}\left(|\Im V|^{2}+\frac{1}{2}\left|B_{\tau}\right|^{2}\right)|\psi|^{2} & \leq b_{3}^{2} \int_{\mathbb{R}^{d}}\left|\nabla_{A} \psi\right|^{2} .
\end{aligned}
$$

Then $\sigma_{\mathrm{p}}\left(H_{A, V}\right)=\varnothing$. 


\section{Absence of eigenvalues: the Birman-Schwinger principle}

The main role in our proof of Theorem 1 is played by the Birman-Schwinger operator

$$
K_{z}:=|V|^{1 / 2}\left(H_{0}-z\right)^{-1} V_{1 / 2} \quad \text { with } \quad V_{1 / 2}:=|V|^{1 / 2} \operatorname{sgn}(V),
$$

where $\operatorname{sgn}(z)$ is the complex signum function defined by $\operatorname{sgn}(z):=z /|z|$ for $z \in \mathbb{C} \backslash\{0\}$ and $\operatorname{sgn}(0):=0$. We abuse the notation by using the same symbols for maximal operators of multiplication and their generating functions. The operator $K_{z}$ is well defined (on its natural domain of the composition of three operators) for all $z \in \mathbb{C}$ and $d \geq 3$.

If $z \notin[0,+\infty)$, however, we have a useful formula for the integral kernel of $K_{z}$ :

$$
K_{z}(x, y)=|V|^{1 / 2}(x) G_{z}(x, y) V_{1 / 2}(y),
$$

where $G_{z}$ is the Green's function of $H_{0}-z$, i.e. the integral kernel of the resolvent $\left(H_{0}-z\right)^{-1}$. We observe that $K_{z}$ is a bounded operator for all $z \notin[0,+\infty)$ and $d \geq 3$ under our hypothesis (11). Indeed, $V_{1 / 2}$ maps $L^{2}\left(\mathbb{R}^{d}\right)$ to $H^{-1}\left(\mathbb{R}^{d}\right)$ by duality, $\left(H_{0}-z\right)^{-1}$ is an isomorphism between $H^{-1}\left(\mathbb{R}^{d}\right)$ and $H^{1}\left(\mathbb{R}^{d}\right)$ and the latter space is mapped by $|V|^{1 / 2}$ back to $L^{2}\left(\mathbb{R}^{d}\right)$.

Moreover, if $d=3$, we have an explicit formula

$$
G_{z}(x, y):=\frac{1}{4 \pi} \frac{e^{-\sqrt{-z}|x-y|}}{|x-y|} .
$$

Here and in the sequel we choose the principal branch of the square root. Using this explicit formula, we are able to show that $K_{z}$ is bounded by $a$ under the hypothesis (1).

Lemma 1. Let $d=3$ and assume (1). Then

$$
\forall z \notin(0,+\infty), \quad\left\|K_{z}\right\| \leq a .
$$

Proof. We start with an equivalent formulation of (1), in any dimension $d \geq 3$. Writing $g:=H_{0}^{1 / 2} \psi$ in (11), we have

$$
\left\||V|^{1 / 2} H_{0}^{-1 / 2} g\right\|^{2} \leq a\left\|\nabla H_{0}^{-1 / 2} g\right\|^{2}=a\|g\|^{2},
$$

where $\|\cdot\|$ denotes the norm in $L^{2}\left(\mathbb{R}^{d}\right)$. Since the range of $H_{0}^{1 / 2}$ is dense in $L^{2}\left(\mathbb{R}^{d}\right)$, we see that (1) is equivalent to

$$
\left\||V|^{1 / 2} H_{0}^{-1 / 2}\right\|^{2} \leq a .
$$

It follows (by taking the adjoint) that also

$$
\left\|H_{0}^{-1 / 2}|V|^{1 / 2}\right\|^{2} \leq a .
$$

Now we assume $d=3$, where the explicit formula (23) for the Green function is available. By virtue of the pointwise bound

$$
\forall z \notin(0,+\infty), \quad \forall x, y \in \mathbb{R}^{3}, \quad\left|G_{z}(x, y)\right| \leq G_{0}(x, y)
$$

we have

$$
\left|\left(f, K_{z} g\right)\right| \leq\left(|f|, \tilde{K}_{0}|g|\right) \leq\left\|\tilde{K}_{0}\right\|\|f\|\|g\|
$$

for every $z \notin(0,+\infty)$ and all $f, g \in L^{2}\left(\mathbb{R}^{3}\right)$, where

$$
\tilde{K}_{0}:=|V|^{1 / 2} H_{0}^{-1}|V|^{1 / 2}
$$

and $(\cdot, \cdot)$ denotes the inner product in $L^{2}\left(\mathbb{R}^{3}\right)$ (conjugate linear in the first argument). Using (25) and (26), we have

$$
\left\|\tilde{K}_{0}\right\|=\left\||V|^{1 / 2} H_{0}^{-1}|V|^{1 / 2}\right\| \leq\left\||V|^{1 / 2} H_{0}^{-1 / 2}\right\|\left\|H_{0}^{-1 / 2}|V|^{1 / 2}\right\| \leq a .
$$

Consequently, (28) and (29) imply (24). 
The following lemma provides an (integral) criterion for the existence of solutions to the (differential) eigenvalue equation of $H_{V}$. It can be considered as a one-sided version of the Birman-Schwinger principle extended to possible eigenvalues in $[0,+\infty)$ as well.

Lemma 2. Let $d=3$ and assume (11). If $H_{V} \psi=\lambda \psi$ with some $\lambda \in \mathbb{C}$ and $\psi \in \mathrm{D}\left(H_{V}\right)$, then $\phi:=|V|^{1 / 2} \psi$ obeys

$$
\forall \varphi \in L^{2}\left(\mathbb{R}^{3}\right), \quad \lim _{\varepsilon \rightarrow 0^{ \pm}}\left(\varphi, K_{\lambda+i \varepsilon} \phi\right)=-(\varphi, \phi) .
$$

Proof. Given any $\lambda \in \mathbb{C}$, there is $\varepsilon_{0}>0$ such that $\lambda+i \varepsilon \notin[0,+\infty)$ for all real $\varepsilon$ satisfying $0<|\varepsilon|<\varepsilon_{0}$. By density of $C_{0}^{\infty}\left(\mathbb{R}^{3}\right)$ in $L^{2}\left(\mathbb{R}^{3}\right)$ and Lemma 1, it is enough to prove (30) for $\varphi \in C_{0}^{\infty}\left(\mathbb{R}^{3}\right)$. We have

$$
\left(\varphi, K_{\lambda+i \varepsilon} \phi\right)=\iint_{\mathbb{R}^{3} \times \mathbb{R}^{3}} \overline{\varphi(x)}|V|^{1 / 2}(x) G_{\lambda+i \varepsilon}(x, y) V(y) \psi(y) d x d y=\int_{\mathbb{R}^{3}} \eta_{\varepsilon}(y) V(y) \psi(y) d y,
$$

where

$$
\eta_{\varepsilon}:=\int_{\mathbb{R}^{3}} \overline{\varphi(x)}|V|^{1 / 2}(x) G_{\lambda+i \varepsilon}(x, \cdot) d x=\left(H_{0}-\lambda-i \varepsilon\right)^{-1}|V|^{1 / 2} \bar{\varphi},
$$

where the second equality holds due to the symmetry $G_{z}(x, y)=G_{z}(y, x)$. In view of (1), $|V|^{1 / 2} \bar{\varphi} \in$ $L^{2}\left(\mathbb{R}^{3}\right)$. Since $\varepsilon \neq 0$ is so small that $\lambda+i \varepsilon \notin \sigma\left(H_{0}\right)$, we have $\eta_{\varepsilon} \in \mathrm{D}\left(H_{0}\right)=H^{2}\left(\mathbb{R}^{3}\right)$. In particular, $\eta_{\varepsilon} \in H^{1}\left(\mathbb{R}^{3}\right)$ and the weak formulation of the eigenvalue equation $H_{V} \psi=\lambda \psi$ yields

$$
\begin{aligned}
\int_{\mathbb{R}^{3}} \eta_{\varepsilon}(y) V(y) \psi(y) d y & =-\left(\nabla \overline{\eta_{\varepsilon}}, \nabla \psi\right)+\lambda\left(\overline{\eta_{\varepsilon}}, \psi\right) \\
& =-\left(\nabla \bar{\psi}, \nabla \eta_{\varepsilon}\right)+\lambda\left(\bar{\psi}, \eta_{\varepsilon}\right) \\
& =-\left(\nabla \bar{\psi}, \nabla \eta_{\varepsilon}\right)+(\lambda+i \varepsilon)\left(\bar{\psi}, \eta_{\varepsilon}\right)-i \varepsilon\left(\bar{\psi}, \eta_{\varepsilon}\right) \\
& =-\left(\bar{\psi},|V|^{1 / 2} \bar{\varphi}\right)-i \varepsilon\left(\bar{\psi}, \eta_{\varepsilon}\right) \\
& =-\left(\varphi,|V|^{1 / 2} \psi\right)-i \varepsilon\left(\overline{\eta_{\varepsilon}}, \psi\right) .
\end{aligned}
$$

Here the last but one equality follows from the weak formulation of the resolvent equation $\left(H_{0}-\lambda-i \varepsilon\right) \eta_{\varepsilon}=$ $|V|^{1 / 2} \bar{\varphi}$. Consequently, (31) and (32) imply (30) after taking the limit $\varepsilon \rightarrow 0^{ \pm}$, provided that $\varepsilon\left(\bar{\eta}_{\varepsilon}, \psi\right) \rightarrow 0$ as $\varepsilon \rightarrow 0$. To see the latter, we write

$$
\left|\left(\overline{\eta_{\varepsilon}}, \psi\right)\right|=\left|\left(\varphi, M_{\varepsilon} \psi\right)\right| \leq\|\varphi\|\left\|M_{\varepsilon}\right\|\|\psi\|,
$$

where $M_{\varepsilon}:=\chi_{\Omega}|V|^{1 / 2}\left(H_{0}-\lambda-i \varepsilon\right)^{-1}$ with $\Omega:=\operatorname{supp} \varphi$, and it remains to show that $\varepsilon\left\|M_{\varepsilon}\right\|$ tends to zero as $\varepsilon \rightarrow 0$. Following [22, Thm. III.6], we use the resolvent kernel (23) and estimate $\left\|M_{\varepsilon}\right\| \leq\left\|M_{\varepsilon}\right\|_{\text {HS }}$. We have

$$
\left\|M_{\varepsilon}\right\|_{\mathrm{HS}}^{2}=\frac{1}{(4 \pi)^{2}} \iint_{\Omega \times \mathbb{R}^{3}}|V(x)| \frac{e^{-2 \kappa(\varepsilon)|x-y|}}{|x-y|^{2}} d x d y=\frac{1}{4 \pi \kappa(\varepsilon)} \int_{\Omega}|V(x)| d x,
$$

where the last integral is bounded because $V \in L_{\text {loc }}^{1}\left(\mathbb{R}^{3}\right)$ as a consequence of (10) and

$$
\kappa(\varepsilon):=\Re \sqrt{-(\lambda+i \varepsilon)} \sim \begin{cases}|\varepsilon|^{1 / 2} & \text { if } \lambda=0, \\ |\varepsilon| & \text { if } \Re \lambda>0 \& \Im \lambda=0, \\ 1 & \text { otherwise. }\end{cases}
$$

Hence, $\varepsilon\left\|M_{\varepsilon}\right\|$ behaves at least as $\mathcal{O}\left(\varepsilon^{1 / 2}\right)$ as $\varepsilon \rightarrow 0$, which concludes the proof of the lemma.

Remark 1. Lemma 2 resembles [22, Thm. III.6] in the self-adjoint case. It is also related to the recent abstract result [13, Prop. 3.1].

Now we are in a position to establish the absence of eigenvalues in three dimensions.

Theorem 5. Let $d=3$ and assume (1). Then $\sigma_{\mathrm{p}}\left(H_{V}\right)=\varnothing$.

Proof. Assume there exists $\lambda \in \mathbb{C}$ and a non-trivial $\psi \in \mathrm{D}\left(H_{V}\right)$ such that $H_{V} \psi=\lambda \psi$. Since the spectrum of $H_{0}$ is purely continuous, the theorem clearly holds for $V=0$ and we may thus suppose that $V$ is non-trivial. But then $\phi:=|V|^{1 / 2} \psi$ is also non-trivial, otherwise $\psi$ would be a non-trivial solution of $H_{0} \psi=\lambda \psi$, which is again impossible by the absence of eigenvalues for $H_{0}$. Now, Lemma 2 with $\varphi:=\phi$ and Lemma 1 yield

$$
a\|\phi\|^{2} \geq \lim _{\varepsilon \rightarrow 0^{ \pm}}\left|\left(\phi, K_{\lambda+i \varepsilon} \phi\right)\right|=\|\phi\|^{2} .
$$

This is a contradiction because $a<1$. 


\section{Absence of the continuous spectrum outside $[0,+\infty)$}

The following lemma is a modification of the idea behind Lemma 2 to deal with the continuous spectrum. We prove it in all dimensions $d \geq 3$.

Lemma 3. Let $d \geq 3$ and assume (11). If $\left\|H_{V} \psi_{n}-\lambda \psi_{n}\right\| \rightarrow 0$ as $n \rightarrow \infty$ with some $\lambda \in \mathbb{C} \backslash \mathbb{R}$ and $\left\{\psi_{n}\right\}_{n \in \mathbb{N}} \subset \mathrm{D}\left(H_{V}\right)$ such that $\left\|\psi_{n}\right\|=1$ for all $n \in \mathbb{N}$, then $\phi_{n}:=|V|^{1 / 2} \psi_{n}$ obeys

$$
\lim _{n \rightarrow \infty} \frac{\left(\phi_{n}, K_{\lambda} \phi_{n}\right)}{\left\|\phi_{n}\right\|^{2}}=-1 \text {. }
$$

Proof. The proof is similar to that of Lemma 2. We have

$$
\left(\phi_{n}, K_{\lambda} \phi_{n}\right)=\int_{\mathbb{R}^{d}} \eta_{n}(y) V(y) \psi_{n}(y) d y=v\left(\overline{\eta_{n}}, \psi_{n}\right),
$$

where $(\cdot, \cdot)$ denotes the inner product in $L^{2}\left(\mathbb{R}^{d}\right)$ and the function

$$
\eta_{n}:=\int_{\mathbb{R}^{d}} \overline{\phi_{n}(x)}|V|^{1 / 2}(x) G_{\lambda}(x, \cdot) d x=\left(H_{0}-\lambda\right)^{-1}|V|^{1 / 2} \overline{\phi_{n}}
$$

belongs to $H^{1}\left(\mathbb{R}^{d}\right)$. Indeed,

$$
\eta_{n}=\left(H_{0}-\lambda\right)^{-1} H_{0}^{1 / 2} H_{0}^{-1 / 2}|V|^{1 / 2} \overline{\phi_{n}},
$$

where $\phi_{n} \in L^{2}\left(\mathbb{R}^{d}\right)$ by (10), $H_{0}^{-1 / 2}|V|^{1 / 2}$ is bounded due to (26) and $\left(H_{0}-\lambda\right)^{-1} H_{0}^{1 / 2}$ maps $L^{2}\left(\mathbb{R}^{d}\right)$ to $H^{1}\left(\mathbb{R}^{d}\right)$. More specifically,

$$
\left\|\eta_{n}\right\| \leq C_{\lambda} \sqrt{a}\left\|\phi_{n}\right\|, \quad \text { where } \quad C_{\lambda}:=\sup _{\xi \in[0, \infty)}\left|\frac{\xi}{\xi^{2}-\lambda}\right| .
$$

In analogy with (32), we are thus allowed to write

$$
\begin{aligned}
v\left(\overline{\eta_{n}}, \psi_{n}\right) & =h_{V}\left(\overline{\eta_{n}}, \psi_{n}\right)-\lambda\left(\overline{\eta_{n}}, \psi_{n}\right)-\left(\nabla \overline{\eta_{n}}, \nabla \psi_{n}\right)+\lambda\left(\overline{\eta_{n}}, \psi_{n}\right) \\
& =\left(\overline{\eta_{n}},\left(H_{V}-\lambda\right) \psi_{n}\right)-h_{0}\left(\overline{\psi_{n}}, \eta_{n}\right)+\lambda\left(\overline{\psi_{n}}, \eta_{n}\right) .
\end{aligned}
$$

By the second representation theorem (cf. [16, Thm. VI.2.23]) and (36),

$$
\begin{aligned}
h_{0}\left(\overline{\psi_{n}}, \eta_{n}\right)-\lambda\left(\overline{\psi_{n}}, \eta_{n}\right) & =\left(H_{0}^{1 / 2} \overline{\psi_{n}}, H_{0}^{1 / 2} \eta_{n}\right)-\lambda\left(\overline{\psi_{n}}, \eta_{n}\right) \\
& =\left(H_{0}^{1 / 2} \overline{\psi_{n}},\left(H_{0}-\lambda+\lambda\right)\left(H_{0}-\lambda\right)^{-1} H_{0}^{-1 / 2}|V|^{1 / 2} \overline{\phi_{n}}\right)-\lambda\left(\overline{\psi_{n}}, \eta_{n}\right) \\
& =\left(H_{0}^{1 / 2} \overline{\psi_{n}}, H_{0}^{-1 / 2}|V|^{1 / 2} \overline{\phi_{n}}\right) \\
& =\left(\left(H_{0}^{-1 / 2}|V|^{1 / 2}\right)^{*} H_{0}^{1 / 2} \overline{\psi_{n}}, \overline{\phi_{n}}\right) \\
& =\left(|V|^{1 / 2} \overline{\psi_{n}}, \overline{\phi_{n}}\right) \\
& =\left\|\phi_{n}\right\|^{2} .
\end{aligned}
$$

Since

$$
\left\|H_{V} \psi_{n}-\lambda \psi_{n}\right\|=\sup _{\substack{\varphi \in L^{2}\left(\mathbb{R}^{d}\right) \\ \varphi \neq 0}} \frac{\left|\left(\varphi, H_{V} \psi_{n}-\lambda \psi_{n}\right)\right|}{\|\varphi\|} \geq\left|\left\|\nabla \psi_{n}\right\|^{2}+v\left[\psi_{n}\right]-\lambda\right|,
$$

where the inequality is obtained by choosing $\varphi:=\psi_{n}$, and the left hand side vanishes as $n \rightarrow \infty$, we see that $\Im v\left[\psi_{n}\right]$ tends to $\Im \lambda \neq 0$ as $n \rightarrow \infty$. In particular,

$$
\liminf _{n \rightarrow \infty}\left\|\phi_{n}\right\|>0 .
$$

Using (39) in (38), recalling (35), dividing the obtained identity by $\left\|\phi_{n}\right\|^{2}$ (which is non-zero for all sufficiently large $n$ due to (40) ) and taking the limit as $n \rightarrow \infty$, we arrive at

$$
\lim _{n \rightarrow \infty} \frac{\left(\phi_{n}, K_{\lambda} \phi_{n}\right)}{\left\|\phi_{n}\right\|^{2}}+1=\lim _{n \rightarrow \infty} \frac{\left(\overline{\eta_{n}},\left(H_{V}-\lambda\right) \psi_{n}\right)}{\left\|\phi_{n}\right\|^{2}} .
$$

In view of (37) and (40), the right hand side equals zero by the hypothesis. 
Now we are in a position to establish the absence of the continuous spectrum outside $[0,+\infty)$.

Theorem 6. Let $d=3$ and assume (1). Then $\sigma_{\mathrm{c}}\left(H_{V}\right) \subset[0,+\infty)$.

Proof. By (1), $\Re h_{V}[\psi] \geq(1-a)\|\nabla \psi\|^{2} \geq 0$ for all $\psi \in H^{1}\left(\mathbb{R}^{3}\right)$. Since $H_{V}$ is m-sectorial, it follows that the spectrum of $H_{V}$ is contained in the right complex half-plane (cf. [16, Thm. V.3.2]). Assume that there exists $\lambda \in \mathbb{C}$ with $\Re \lambda \geq 0$ and $\Im \lambda \neq 0$ such that $\lambda \in \sigma_{\mathrm{c}}\left(H_{V}\right)$. Then $\lambda$ belongs to the kind of essential spectrum which is characterised by the existence of a singular sequence of $H_{V}$ corresponding to $\lambda$ (cf. [8, Thm. IX.1.3]): $\exists\left\{\psi_{n}\right\}_{n \in \mathbb{N}} \subset \mathrm{D}\left(H_{V}\right),\left\|\psi_{n}\right\|=1$ for all $n \in \mathbb{N},\left\|\left(H_{V}-\lambda\right) \psi_{n}\right\| \rightarrow 0$ as $n \rightarrow \infty$ and $\left\{\psi_{n}\right\}_{n \in \mathbb{N}}$ is weakly converging to zero. By Lemma 3 and Lemma 1 .

$$
a \geq\left\|K_{\lambda}\right\| \geq\left|\lim _{n \rightarrow \infty} \frac{\left(\phi_{n}, K_{\lambda} \phi_{n}\right)}{\left\|\phi_{n}\right\|^{2}}\right|=1,
$$

This is a contradiction because $a<1$.

We remark that the last step of the proof of Theorem 6 requires Lemma 1 for which $d=3$ is crucial.

\section{Inclusion of the spectrum in $[0,+\infty)$}

The opposite inclusion follows by an explicit construction of a singular sequence of $H_{V}$ corresponding to non-negative energies. Since the operator $H_{V}$ is defined through its sesquilinear form, it is convenient to have a criterion which requires that the singular sequence is in the form domain only. Unable to find a reference in the general case, we state the abstract version first (for the self-adjoint situation, see [18, Thm. 5]).

Lemma 4. Let $H$ be an m-sectorial accretive operator in a complex Hilbert space $\mathcal{H}$ which is associated with a (densely defined, closed, sectorial) sesquilinear form $h$. Given $\lambda \in \mathbb{C}$, assume that there exists a sequence $\left\{\phi_{n}\right\}_{n \in \mathbb{N}} \subset \mathrm{D}(h)$ such that $\left\|\phi_{n}\right\|=1$ for all $n \in \mathbb{N}$ and

$$
\sup _{\substack{\psi \in \mathrm{D}(h) \\ \psi \neq 0}} \frac{\left|h\left(\phi_{n}, \psi\right)-\lambda\left(\phi_{n}, \psi\right)\right|}{\|\psi\|_{\mathrm{D}(h)}} \underset{n \rightarrow \infty}{\longrightarrow} 0
$$

where $\|\psi\|_{\mathrm{D}(h)}:=\sqrt{\Re h[\psi]+\|\psi\|^{2}}$. Then $\lambda \in \sigma(H)$.

Remark 2. Notice that the left hand side of (41) is the norm of the vector $H^{*} \phi_{n}-\bar{\lambda} \phi_{n}$ in the dual space $\mathrm{D}(h)^{*}$, when $\mathrm{D}(h)$ is thought as the subspace of $\mathcal{H}$ equipped with the norm $\|\cdot\|_{\mathrm{D}(h)}$.

Proof. We proceed by contradiction: Assume the hypotheses of the theorem and $\lambda \notin \sigma(H)$. The latter means that for every $g \in \mathcal{H}$ there exists $\psi \in \mathrm{D}(H)$ such that $H \psi-\lambda \psi=g$. That is, $\psi=(H-\lambda)^{-1} g$ and $(H-\lambda)^{-1}$ is bounded as an operator on $\mathcal{H}$ onto $\mathcal{H}$. The weak formulation of the resolvent equation reads

$$
\forall \phi \in \mathrm{D}(h), \quad h(\phi, \psi)-\lambda(\phi, \psi)=(\phi, g) .
$$

Consequently, for every $\phi \in \mathrm{D}(h)$,

$$
C_{\lambda} \sup _{\substack{\psi \in \mathrm{D}(h) \\ \psi \neq 0}} \frac{|h(\phi, \psi)-\lambda(\phi, \psi)|}{\|\psi\|_{\mathrm{D}(h)}} \geq \sup _{\substack{g \in \mathcal{H} \\ g \neq 0}} \frac{|h(\phi, \psi)-\lambda(\phi, \psi)|}{\|g\|}=\sup _{\substack{g \in \mathcal{H} \\ g \neq 0}} \frac{|(\phi, g)|}{\|g\|}=\|\phi\|,
$$

where $\psi$ and $g$ are related through (42) and the constant

$$
C_{\lambda}:=\sup _{\substack{g \in \mathcal{H} \\ g \neq 0}} \frac{\|\psi\|_{\mathrm{D}(h)}}{\|g\|}
$$

is finite because the resolvent $(H-\lambda)^{-1}$ maps $\mathcal{H}$ onto $\mathrm{D}(H) \subset \mathrm{D}(h)$. More specifically,

$$
\begin{aligned}
\|\psi\|_{\mathrm{D}(h)}^{2} & =\Re h\left[(H-\lambda)^{-1} g\right]+\left\|(H-\lambda)^{-1} g\right\|^{2} \\
& =\Re\left((H-\lambda)^{-1} g, H(H-\lambda)^{-1} g\right)+\left\|(H-\lambda)^{-1} g\right\|^{2} \\
& \leq\left(\left\|(H-\lambda)^{-1}\right\|\left\|H(H-\lambda)^{-1}\right\|+\left\|(H-\lambda)^{-1}\right\|^{2}\right)\|g\|^{2} .
\end{aligned}
$$


Choosing $\phi:=\phi_{n}$ in (43), we get that the left hand side tends to zero as $n \rightarrow \infty$ by (41), while the right hand side equals one due to the normalisation of $\left\{\phi_{n}\right\}_{n \in \mathbb{N}}$, a contradiction.

Now we are in a position to prove the inclusion of the interval $[0,+\infty)$ in the spectrum of $H_{V}$. The following result holds in all dimensions $d \geq 3$.

Theorem 7. Let $d \geq 3$ and assume (11). Then $\sigma\left(H_{V}\right) \supset[0,+\infty)$.

Proof. We construct the sequence $\left\{\phi_{n}\right\}_{n \in \mathbb{N}}$ from Lemma 4 applied to $H_{V}$ by setting

$$
\phi_{n}(x):=\varphi_{n}(x) e^{i k \cdot x},
$$

where $k \in \mathbb{R}^{d}$ is such that $|k|^{2}=\lambda \in[0,+\infty), \varphi_{n}(x):=n^{-d / 2} \varphi_{1}(x / n)$ for all $n \in \mathbb{N}$ (with the convention $0 \notin \mathbb{N})$ and $\varphi_{1} \in C_{0}^{\infty}\left(\mathbb{R}^{d}\right)$ is a function such that $\left\|\varphi_{1}\right\|=1$. The normalisation factor in the definition of $\varphi_{n}$ is chosen in such a way that

$$
\left\|\varphi_{n}\right\|=\left\|\varphi_{1}\right\|=1, \quad\left\|\nabla \varphi_{n}\right\|=n^{-1}\left\|\nabla \varphi_{1}\right\|, \quad\left\|\Delta \varphi_{n}\right\|=n^{-2}\left\|\Delta \varphi_{1}\right\|
$$

for all $n \in \mathbb{N}$. Then $\left\|\phi_{n}\right\|=1$ and $\phi_{n} \in \mathrm{D}\left(h_{V}\right)=\mathrm{D}\left(h_{0}\right)=H^{1}\left(\mathbb{R}^{d}\right)$ for all $n \in \mathbb{N}$. Furthermore,

$$
\left\|-\Delta \phi_{n}-\lambda \phi_{n}\right\|=\left\|-\Delta \varphi_{n}+2 i k \cdot \nabla \varphi_{n}\right\| \leq\left\|\Delta \varphi_{n}\right\|+2|k|\left\|\nabla \varphi_{n}\right\| \underset{n \rightarrow \infty}{\longrightarrow} 0 .
$$

In fact, $\left\{\phi_{n}\right\}_{n \in \mathbb{N}}$ is the usual singular sequence of $H_{0}$ corresponding to $\lambda$. At the same time,

$$
\left|v\left[\phi_{n}\right]\right| \leq\left\||V|^{1 / 2} \varphi_{n}\right\|^{2} \leq a\left\|\nabla \varphi_{n}\right\|^{2} \underset{n \rightarrow \infty}{\longrightarrow} 0,
$$

where the second inequality follows by (1).

The numerator in (41) can be estimated as follows

$$
\begin{aligned}
\left|h_{V}\left(\phi_{n}, \psi\right)-\lambda\left(\phi_{n}, \psi\right)\right| & =\left|\left(-\Delta \phi_{n}-\lambda \phi_{n}, \psi\right)+v\left(\phi_{n}, \psi\right)\right| \\
& \leq\left\|-\Delta \phi_{n}-\lambda \phi_{n}\right\|\|\psi\|+\sqrt{\left|v\left[\phi_{n}\right]\right|} \sqrt{|v[\psi]|} \\
& \leq\left\|-\Delta \phi_{n}-\lambda \phi_{n}\right\|\|\psi\|+\sqrt{\left|v\left[\phi_{n}\right]\right|} \sqrt{a}\|\nabla \psi\|,
\end{aligned}
$$

where the last inequality is due to (11). As for the denominator in (41), employing (11) again, we have

$$
\|\psi\|_{\mathrm{D}(h)}^{2}=\|\nabla \psi\|^{2}+\Re v[\psi]+\|\psi\|^{2} \geq(1-a)\|\nabla \psi\|^{2}+\|\psi\|^{2} \geq(1-a)\|\psi\|_{\mathrm{D}\left(h_{0}\right)}^{2},
$$

where $\|\cdot\|_{\mathrm{D}\left(h_{0}\right)}$ is just the usual norm of $H^{1}\left(\mathbb{R}^{d}\right)$. Putting these estimates together, we have the bound

$$
\sup _{\substack{\psi \in \mathrm{D}\left(h_{V}\right) \\ \psi \neq 0}} \frac{\left|h_{V}\left(\phi_{n}, \psi\right)-\lambda\left(\phi_{n}, \psi\right)\right|}{\|\psi\|_{\mathrm{D}\left(h_{V}\right)}} \leq \frac{\left\|-\Delta \phi_{n}-\lambda \phi_{n}\right\|+\sqrt{\left|v\left[\phi_{n}\right]\right|} \sqrt{a}}{\sqrt{1-a}},
$$

where the right hand side tends to zero due to (44) and (45).

Summing up, given $\lambda \in[0,+\infty)$, we have shown that the sequence $\left\{\phi_{n}\right\}_{n \in \mathbb{N}}$ satisfies all the hypotheses of Lemma 4. Consequently, $[0,+\infty) \subset \sigma\left(H_{V}\right)$.

Proof of Theorem 1. To conclude, Theorem 1 follows as a consequence of Theorems 5, 6, 7 and the absence of the residual spectrum justified already in Section 1.

\section{Absence of eigenvalues: the method of multipliers}

In this last section, we prove Theorems 2 , 3 and 4 by a completely different approach in comparison with the previous sections. Namely, we extend the method of multipliers developed in the self-adjoint context in [2] to complex-valued potentials. Here we proceed in all dimensions $d \geq 3$.

Let us consider the equation

$$
\Delta u+\lambda u=f
$$


where $\lambda$ is any complex constant; we write $\lambda_{1}:=\Re \lambda$ and $\lambda_{2}:=\Im \lambda$. Given a measurable function $f: \mathbb{R}^{d} \rightarrow \mathbb{C}$ that we assume to merely belong to $H^{-1}\left(\mathbb{R}^{d}\right)$, we say that $u$ is a solution of (46) if $u \in H^{1}\left(\mathbb{R}^{d}\right)$ and

$$
\forall v \in H^{1}\left(\mathbb{R}^{d}\right), \quad-(\nabla v, \nabla u)+\lambda(v, u)=(v, f) .
$$

Here, with an abuse of notation, the same symbol $(\cdot, \cdot)$ is used for the inner product in $L^{2}\left(\mathbb{R}^{d}\right)$ and for the duality pairing between $H^{1}\left(\mathbb{R}^{d}\right)$ and $H^{-1}\left(\mathbb{R}^{d}\right)$ on the left and right hand side of (47), respectively. Equation (46) is related to the eigenvalue problem of $H_{V}$ by setting $f:=V u$. Notice that any eigenvalue $\lambda$ of $H_{V}$ necessarily satisfies $\lambda_{1}>0$ due to (11). If $u$ is a solution of (46), we set

$$
u^{ \pm}(x):=e^{ \pm i \operatorname{sgn}\left(\lambda_{2}\right) \lambda_{1}^{\frac{1}{2}}|x|} u(x), \quad \operatorname{sgn}\left(\lambda_{2}\right):=\left\{\begin{array}{lll}
\frac{\lambda_{2}}{\left|\lambda_{2}\right|} & \text { if } & \lambda_{2} \neq 0 \\
1 & \text { if } & \lambda_{2}=0
\end{array}\right.
$$

In order to prove Theorem 2, we establish the following result, which shows that (46) has no nontrivial solutions provided that $f$ is small in a suitable sense.

Theorem 8. Let $d \geq 3$. Let $u$ be a solution of (46) with $\Re \lambda>0$, and assume that $f$ satisfies

$$
\|x f\| \leq \Lambda\left\|\nabla u^{-}\right\|, \quad\|x f\| \leq \Lambda\|\nabla u\|
$$

where $\Lambda$ is determined by

$$
\frac{2(2 d-3)}{d-2} \Lambda+\frac{\sqrt{2}}{\sqrt{d-2}} \Lambda^{\frac{3}{2}}<1 .
$$

Then $u=0$.

Proof. The proof relies on direct techniques, based on multiplication and integration by parts, inspired by [2], in which the methods by [5, 14] are developed and refined. Here we propose some slight modifications in the arguments, essentially due to the fact that we need to handle complex-valued potentials. To save space, we abbreviate $\int:=\int_{\mathbb{R}^{d}}$ and omit arguments of integrated functions.

Following [2, we divide the proof into two cases: $\left|\lambda_{2}\right| \leq \lambda_{1}$ and $\left|\lambda_{2}\right|>\lambda_{1}$.

Case $\left|\lambda_{2}\right| \leq \lambda_{1}$. Our first step consists in approximating solutions of (47) by a standard cutoff and mollification argument, which is fundamental to make rigorous the proof in the sequel. To this aim, let $\xi_{R}: \mathbb{R}^{d} \rightarrow[0,1]$ be a smooth function such that

$$
\xi=1 \text { in } B_{R}, \quad \xi=0 \text { in } \mathbb{R}^{d} \backslash B_{2 R}, \quad\left|\nabla \xi_{R}\right| \leq 2 R^{-1}, \quad\left|\Delta \xi_{R}\right| \leq 2 R^{-1}|x|^{-1},
$$

for any $R>0$ sufficiently large, where $B_{R}:=\{|x|<R\}$. For a function $g: \mathbb{R}^{d} \rightarrow \mathbb{C}$, we then denote $g_{R}:=g \xi_{R}$. If $u \in H^{1}\left(\mathbb{R}^{d}\right)$ is a solution to (47), we see that $u_{R} \in H^{1}\left(\mathbb{R}^{d}\right)$ solves

$$
\Delta u_{R}+\lambda u_{R}=f_{R}-2 \nabla \xi_{R} \cdot \nabla u-u \Delta \xi_{R}=: \widetilde{f}_{R}
$$

in the weak sense of (47). Notice that, since $f$ satisfies conditions (49) and (50), we have

$$
\left\|x \widetilde{f}_{R}\right\| \leq \Lambda\left\|\nabla u_{R}^{-}\right\|+\epsilon^{2}(R), \quad\left\|x \widetilde{f}_{R}\right\| \leq \Lambda\left\|\nabla u_{R}\right\|+\epsilon^{2}(R), \quad \lim _{R \rightarrow \infty} \epsilon^{2}(R)=0 .
$$

Indeed, by (51),

$$
\left\|x \widetilde{f}_{R}\right\| \leq\left\|x f_{R}\right\|+8\left(\int_{R<|x|<2 R}|\nabla u|^{2}\right)^{\frac{1}{2}}+4 R^{-2}\left(\int_{R<|x|<2 R}|u|^{2}\right)^{\frac{1}{2}},
$$

where the last two terms tends to 0 as $R \rightarrow \infty$, since $u \in H^{1}\left(\mathbb{R}^{d}\right)$.

Let now $\phi \in C_{0}^{\infty}\left(\mathbb{R}^{d}\right)$ be a function such that $\int \phi=1$, and define, for any $\delta>0, \phi_{\delta}(x):=\delta^{-d} \phi\left(\frac{x}{\delta}\right)$. If $u \in H^{1}\left(\mathbb{R}^{d}\right)$ is a solution to (47), we see that $u_{R, \delta}:=u_{R} * \phi_{\delta}$ solves

$$
\Delta u_{R, \delta}+\lambda u_{R, \delta}=\widetilde{f}_{R, \delta}
$$


in the weak sense of (47), where $\widetilde{f}_{R, \delta}:=\widetilde{f}_{R} * \phi_{\delta}$. More specifically,

$$
\forall v \in H^{1}\left(\mathbb{R}^{d}\right), \quad\left(-\nabla v, \nabla u_{R, \delta}\right)+\lambda\left(v, u_{R, \delta}\right)=\left(v, \widetilde{f}_{R, \delta}\right) .
$$

By (53), it turns out that

$$
\left\|x \widetilde{f}_{R, \delta}\right\| \leq \Lambda\left\|\nabla u_{R, \delta}^{-}\right\|+\epsilon^{2}(R), \quad\left\|x \widetilde{f}_{R, \delta}\right\| \leq \Lambda\left\|\nabla u_{R, \delta}\right\|+\epsilon^{2}(R), \quad \lim _{R \rightarrow \infty} \epsilon^{2}(R)=0,
$$

where $u_{R, \delta}^{-}:=u_{R}^{-} * \phi_{\delta}$ and $\Lambda$ as in (50).

We can now start with suitable algebraic manipulations of equation (54), which suitably approximates (47). Let $G_{1}, G_{2}, G_{3}: \mathbb{R}^{d} \rightarrow \mathbb{R}$ be three smooth functions. Choosing $v:=G_{1} u_{R, \delta}$ in (54), integrating by parts and taking the real part of the resulting identity, we arrive at the identity

$$
\lambda_{1} \int G_{1}\left|u_{R, \delta}\right|^{2}-\int G_{1}\left|\nabla u_{R, \delta}\right|^{2}+\frac{1}{2} \int \Delta G_{1}\left|u_{R, \delta}\right|^{2}=\Re \int \tilde{f}_{R, \delta} G_{1} \overline{u_{R, \delta}} .
$$

Analogously, choosing $v:=G_{2} u$ in (47) and taking the imaginary part of the resulting identity, we obtain

$$
\lambda_{2} \int G_{2}\left|u_{R, \delta}\right|^{2}-\Im \int \nabla G_{2} \cdot \overline{u_{R, \delta}} \nabla u_{R, \delta}=\Im \int \widetilde{f}_{R, \delta} G_{2} \overline{u_{R, \delta}},
$$

where the dot denotes the scalar product in $\mathbb{R}^{d}$. Finally, choosing $v:=2 \nabla G_{3} \cdot \nabla u_{R, \delta}+\Delta G_{3} u_{R, \delta}$ in (47), integrating by parts and taking the real part of the resulting identity, we get

$$
\begin{aligned}
\int & \nabla u_{R, \delta} \cdot \nabla^{2} G_{3} \cdot \nabla \overline{u_{R, \delta}}-\frac{1}{4} \int \Delta^{2} G_{3}\left|u_{R, \delta}\right|^{2}+\lambda_{2} \Im \int \nabla G_{3} \cdot u_{R, \delta} \nabla \overline{u_{R, \delta}} \\
& =-\frac{1}{2} \Re \int \widetilde{f}_{R, \delta} \Delta G_{3} \overline{u_{R, \delta}}-\Re \int \widetilde{f}_{R, \delta} \nabla G_{3} \cdot \nabla \overline{u_{R, \delta}},
\end{aligned}
$$

where $\nabla^{2} G_{3}$ denotes the Hessian matrix of $G_{3}$ and $\Delta^{2}:=\Delta \Delta$ is the bi-Laplacian. Notice that identities (56), (57), (58) are justified, since $u_{R, \delta} \in C_{0}^{\infty}\left(\mathbb{R}^{d}\right)$ and $G_{1}, G_{2}, G_{3}$ are smooth, therefore bounded, together with their derivatives of any order, inside the support of $u_{R, \delta}$.

In the following, we assume that $G_{1}, G_{2}, G_{3}$ are radial, i.e. there exist smooth functions $g_{1}, g_{2}, g_{3}$ : $[0, \infty) \rightarrow \mathbb{R}$ such that $G_{i}(x)=g_{i}(|x|)$ for all $x \in \mathbb{R}^{d}$ and $i \in\{1,2,3\}$. Then

$$
\nabla G_{i}(x)=g_{i}^{\prime}(|x|) \frac{x}{|x|}, \quad \Delta G_{i}(x)=g_{i}^{\prime \prime}(|x|)+g_{i}^{\prime}(|x|) \frac{d-1}{|x|}, \quad \nabla^{2} G_{i}(x)=g_{i}^{\prime \prime}(|x|) \frac{x x}{|x|^{2}}+\frac{g_{i}^{\prime}(|x|)}{|x|}\left(I-\frac{x x}{|x|^{2}}\right),
$$

where $I$ denotes the identity on $\mathbb{R}^{d}$ and $x x$ is the dyadic product of $x$ and $x$. For any $g: \mathbb{R}^{d} \rightarrow \mathbb{C}$, denote by

$$
\partial_{r} g(x):=\frac{x}{|x|} \cdot \nabla g(x) \quad \text { and } \quad \nabla_{\tau} g(x):=\left(I-\frac{x x}{|x|^{2}}\right) \cdot \nabla g(x)
$$

the radial derivative and the angular gradient of $g$, respectively, so that $|\nabla g|^{2}=\left|\partial_{r} g\right|^{2}+\left|\nabla_{\tau} g\right|^{2}$.

Taking the sum (56) $+\lambda_{1}^{\frac{1}{2}}(\underline{57})+(58)$, we obtain

$$
\begin{aligned}
& \int\left|\partial_{r} u_{R, \delta}\right|^{2}\left(g_{3}^{\prime \prime}-g_{1}\right)+\int\left|\nabla_{\tau} u_{R, \delta}\right|^{2}\left(\frac{g_{3}^{\prime}}{|x|}-g_{1}\right)+\int\left|u_{R, \delta}\right|^{2}\left(\lambda_{1} g_{1}+\lambda_{2} \lambda_{1}^{\frac{1}{2}} g_{2}\right) \\
& \quad+\int\left|u_{R, \delta}\right|^{2}\left(\frac{1}{2} \Delta G_{1}-\frac{1}{4} \Delta^{2} G_{3}\right)-\lambda_{1}^{\frac{1}{2}} \Im \int \overline{u_{R, \delta}} \nabla u_{R, \delta} \cdot \nabla G_{2}+\lambda_{2} \Im \int u_{R, \delta} \nabla \overline{u_{R, \delta}} \cdot \nabla G_{3} \\
& =\Re \int \widetilde{f}_{R, \delta} G_{1} \overline{u_{R, \delta}}+\lambda^{\frac{1}{2}} \Im \int \widetilde{f}_{R, \delta} G_{2} \overline{u_{R, \delta}}-\frac{1}{2} \Re \int \widetilde{f}_{R, \delta} \overline{u_{R, \delta}} \Delta G_{3}-\Re \int \widetilde{f}_{R, \delta} \nabla \overline{u_{R, \delta}} \cdot \nabla G_{3} .
\end{aligned}
$$

Choosing $g_{1}:=\frac{1}{2} g_{3}^{\prime \prime}$ and $g_{2}:=\operatorname{sgn}\left(\lambda_{2}\right) g_{3}^{\prime}$, the last identity becomes

$$
\begin{aligned}
& \frac{1}{2} \int g_{3}^{\prime \prime}\left(\left|\partial_{r} u_{R, \delta}\right|^{2}+\lambda_{1}\left|u_{R, \delta}\right|^{2}\right)-\operatorname{sgn}\left(\lambda_{2}\right) \lambda_{1}^{\frac{1}{2}} \Im \int g_{3}^{\prime \prime} \overline{u_{R, \delta}} \partial_{r} u_{R, \delta}+\int\left|\nabla_{\tau} u_{R, \delta}\right|^{2}\left(\frac{g_{3}^{\prime}}{r}-\frac{g_{3}^{\prime \prime}}{2}\right) \\
& \quad+\frac{1}{4} \int\left|u_{R, \delta}\right|^{2}\left(\Delta G_{3}^{\prime \prime}-\Delta^{2} G_{3}\right)+\left|\lambda_{2}\right| \lambda_{1}^{\frac{1}{2}} \int g_{3}^{\prime}\left|u_{R, \delta}\right|^{2}+\lambda_{2} \Im \int g_{3}^{\prime} u_{R, \delta} \partial_{r} \overline{u_{R, \delta}} \\
& =\frac{1}{2} \Re \int \widetilde{f}_{R, \delta} g_{3}^{\prime \prime} \overline{u_{R, \delta}}+\lambda_{1}^{\frac{1}{2}} \operatorname{sgn}\left(\lambda_{2}\right) \Im \int \widetilde{f}_{R, \delta} g_{3}^{\prime} \overline{u_{R, \delta}}-\frac{1}{2} \Re \int \widetilde{f}_{R, \delta} \overline{u_{R, \delta}} \Delta G_{3}-\Re \int \widetilde{f}_{R, \delta} \nabla \overline{u_{R, \delta}} \cdot \nabla G_{3},
\end{aligned}
$$


where $G_{3}^{\prime \prime}(x):=g_{3}^{\prime \prime}(|x|)$. Choosing now $G_{3}(x):=|x|^{2}$, the tangential and radial derivatives of $u$ sum up and we obtain

$$
\begin{aligned}
& \int\left(\left|\nabla u_{R, \delta}\right|^{2}+\lambda_{1}\left|u_{R, \delta}\right|^{2}\right)-2 \operatorname{sgn}\left(\lambda_{2}\right) \lambda_{1}^{\frac{1}{2}} \Im \int \overline{u_{R, \delta}} \partial_{r} u_{R, \delta}+2\left|\lambda_{2}\right| \lambda_{1}^{\frac{1}{2}} \int|x|\left|u_{R, \delta}\right|^{2}+2 \lambda_{2} \Im \int|x| u_{R, \delta} \partial_{r} \overline{u_{R, \delta}} \\
& =(1-d) \Re \int \widetilde{f}_{R, \delta} \overline{u_{R, \delta}}+2 \lambda_{1}^{\frac{1}{2}} \operatorname{sgn}\left(\lambda_{2}\right) \Im \int \widetilde{f}_{R, \delta}|x| \overline{u_{R, \delta}}-2 \Re \int \widetilde{f}_{R, \delta} x \cdot \nabla \overline{u_{R, \delta}} .
\end{aligned}
$$

Using

$$
\left|\nabla u_{R, \delta}^{-}\right|^{2}=\left|\nabla u_{R, \delta}-i \operatorname{sgn}\left(\lambda_{2}\right) \lambda_{1}^{\frac{1}{2}} \frac{x}{|x|} u_{R, \delta}\right|^{2}=\left|\nabla u_{R, \delta}\right|^{2}+\lambda_{1}\left|u_{R, \delta}\right|^{2}-2 \operatorname{sgn}\left(\lambda_{2}\right) \lambda_{1}^{\frac{1}{2}} \Im\left(\overline{u_{R, \delta}} \partial_{r} u_{R, \delta}\right),
$$

we can rewrite (60) as follows:

$$
\begin{aligned}
& \int\left|\nabla u_{R, \delta}^{-}\right|^{2}+2\left|\lambda_{2}\right| \lambda_{1}^{\frac{1}{2}} \int|x|\left|u_{R, \delta}\right|^{2}+2 \lambda_{2} \Im \int|x| u_{R, \delta} \partial_{r} \overline{u_{R, \delta}} \\
& =(1-d) \Re \int \tilde{f}_{R, \delta} \overline{u_{R, \delta}}+2 \lambda_{1}^{\frac{1}{2}} \operatorname{sgn}\left(\lambda_{2}\right) \Im \int \tilde{f}_{R, \delta}|x| \overline{u_{R, \delta}}-2 \Re \int \tilde{f}_{R, \delta} x \cdot \nabla \bar{u}_{R, \delta} .
\end{aligned}
$$

Subtracting from the last identity equation (56) with the choice $G_{1}(x):=\left|\lambda_{2}\right| \lambda_{1}^{-\frac{1}{2}}|x|$, we arrive at

$$
\begin{aligned}
& \int\left|\nabla u_{R, \delta}^{-}\right|^{2}-\frac{(d-1)}{2}\left|\lambda_{2}\right| \lambda_{1}^{-\frac{1}{2}} \int \frac{\left|u_{R, \delta}\right|^{2}}{|x|}+\left|\lambda_{2}\right| \lambda_{1}^{\frac{1}{2}} \int|x|\left|u_{R, \delta}\right|^{2} \\
& \quad+\left|\lambda_{2}\right| \lambda_{1}^{-\frac{1}{2}} \int|x|\left|\nabla u_{R, \delta}\right|^{2}+2 \lambda_{2} \Im \int|x| u_{R, \delta} \partial_{r} \overline{u_{R, \delta}} \\
& =(1-d) \Re \int \widetilde{f}_{R, \delta} \bar{u}_{R, \delta}+2 \lambda_{1}^{\frac{1}{2}} \operatorname{sgn}\left(\lambda_{2}\right) \Im \int \widetilde{f}_{R, \delta}|x| \overline{u_{R, \delta}}-2 \Re \int \widetilde{f}_{R, \delta} x \cdot \nabla \overline{u_{R, \delta}}-\left|\lambda_{2}\right| \lambda_{1}^{-\frac{1}{2}} \Re \int \widetilde{f}_{R, \delta}|x| \overline{u_{R, \delta}} .
\end{aligned}
$$

Using (61) again, we obtain the key identity

$$
\begin{aligned}
I:= & \int\left|\nabla u_{R, \delta}^{-}\right|^{2}+\frac{\left|\lambda_{2}\right|}{\lambda_{1}^{\frac{1}{2}}} \int|x|\left|\nabla u_{R, \delta}^{-}\right|^{2}-\frac{(d-1)}{2} \frac{\left|\lambda_{2}\right|}{\lambda_{1}^{\frac{1}{2}}} \int \frac{\left|u_{R, \delta}\right|^{2}}{|x|} \\
& =\underbrace{(1-d) \Re \int \widetilde{f}_{R, \delta} \overline{u_{R, \delta}}}_{I_{1}} \underbrace{-2 \Re \int|x| \widetilde{f}_{R, \delta}\left(\partial_{r} \overline{u_{R, \delta}}+i \operatorname{sgn}\left(\lambda_{2}\right) \lambda_{1}^{\frac{1}{2}} \overline{u_{R, \delta}}\right)}_{I_{2}} \underbrace{-\frac{\lambda_{2}}{\lambda_{1}^{\frac{1}{2}}} \int|x| \widetilde{f}_{R, \delta} \overline{u_{R, \delta}}}_{I_{3}} .
\end{aligned}
$$

By the weighted Hardy inequality

$$
\forall \psi \in C_{0}^{\infty}\left(\mathbb{R}^{d}\right), \quad \int \frac{|\psi|^{2}}{|x|} \leq \frac{4}{(d-1)^{2}} \int|x||\nabla \psi|^{2},
$$

and the facts that $u_{R, \delta} \in C_{0}^{\infty}\left(\mathbb{R}^{d}\right)$ and $\left|u_{R, \delta}\right|=\left|u_{R, \delta}^{-}\right|$, we easily bound the left hand side of (62) from below by a positive quantity as follows

$$
I \geq \int\left|\nabla u_{R, \delta}^{-}\right|^{2}+\frac{\left|\lambda_{2}\right|}{\lambda_{1}^{\frac{1}{2}}} \frac{d-3}{d-1} \int|x|\left|\nabla u_{R, \delta}^{-}\right|^{2} .
$$

We proceed by estimating the individual terms on the right hand side of (62) by means of $\left\|\nabla u_{R, \delta}^{-}\right\|^{2}$. By the Schwarz inequality, the Hardy inequality (6) and thanks to (55), we have

$$
\left|I_{1}\right| \leq(d-1)\left\|x \widetilde{f}_{R, \delta}\right\|\left\|\frac{u_{R, \delta}}{|x|}\right\|=(d-1)\left\|x \widetilde{f}_{R, \delta}\right\|\left\|\frac{u_{R, \delta}^{-}}{|x|}\right\| \leq \frac{2(d-1)}{d-2}\left(\Lambda\left\|\nabla u_{R, \delta}^{-}\right\|^{2}+\epsilon^{2}(R)\left\|\nabla u_{R, \delta}^{-}\right\|\right) .
$$

Since $\partial_{r} \bar{u}_{R, \delta}+i \lambda_{1}^{\frac{1}{2}} \operatorname{sgn}\left(\lambda_{2}\right) \overline{u_{R, \delta}}=\overline{\partial_{r} u_{R, \delta}^{-}}$, we may write

$$
\left|I_{2}\right| \leq 2\left\|x \widetilde{f}_{R, \delta}\right\|\left\|\partial_{r} u_{R, \delta}^{-}\right\| \leq 2\left\|x \widetilde{f}_{R, \delta}\right\|\left\|\nabla u_{R, \delta}^{-}\right\| \leq 2\left(\Lambda\left\|\nabla u_{R, \delta}^{-}\right\|^{2}+\epsilon^{2}(R)\left\|\nabla u_{R, \delta}^{-}\right\|\right) .
$$


If $\lambda_{2} \neq 0$, we also need to estimate the term $I_{3}$. First notice that identity (57) with the constant choice $G_{2}(x):=\frac{\lambda_{2}}{\left|\lambda_{2}\right|}$, immediately gives the $L^{2}$-bound

$$
\left\|u_{R, \delta}\right\|^{2} \leq\left|\lambda_{2}\right|^{-1} \int\left|\widetilde{f}_{R, \delta}\right|\left|u_{R, \delta}\right|
$$

As a consequence, since $\left|\lambda_{2}\right| \leq \lambda_{1}$, we have

$$
\begin{aligned}
\left|I_{3}\right| & \leq \frac{\left|\lambda_{2}\right|}{\lambda_{1}^{\frac{1}{2}}}\left\|x \widetilde{f}_{R, \delta}\right\|\left\|u_{R, \delta}\right\| \leq\left(\Lambda\left\|\nabla u_{R, \delta}^{-}\right\|+\epsilon^{2}(R)\right) \sqrt{\int\left|\widetilde{f}_{R, \delta} \| u_{R, \delta}\right|} \\
& \leq\left(\Lambda\left\|\nabla u_{R, \delta}^{-}\right\|+\epsilon^{2}(R)\right)\left\|x \widetilde{f}_{R, \delta}\right\|^{\frac{1}{2}}\left\|\frac{u_{R, \delta}}{|x|}\right\|^{\frac{1}{2}} \\
& \leq \Lambda^{\frac{3}{2}} \frac{\sqrt{2}}{\sqrt{d-2}}\left\|\nabla u_{R, \delta}^{-}\right\|^{2}+\epsilon^{2}(R) \frac{\sqrt{2}}{\sqrt{d-2}}\left\|\nabla u_{R, \delta}^{-}\right\|\left(\Lambda^{\frac{1}{2}}\left\|\nabla u_{R, \delta}^{-}\right\|+\epsilon(R)\right) .
\end{aligned}
$$

Applying the estimates (64), (65), (66) and (67) in (62), we obtain

$$
\begin{aligned}
& \left(1-\frac{2(2 d-3)}{d-2} \Lambda-\frac{\sqrt{2}}{\sqrt{d-2}} \Lambda^{\frac{3}{2}}\right) \int\left|\nabla u_{R, \delta}^{-}\right|^{2}+\frac{\left|\lambda_{2}\right|}{\lambda_{1}^{\frac{1}{2}}} \frac{d-3}{d-1} \int|x|\left|\nabla u_{R, \delta}^{-}\right|^{2} \\
& \leq \epsilon^{2}(R)\left\|\nabla u_{R, \delta}^{-}\right\|\left(\frac{4 d-6}{d-2}+\frac{\sqrt{2}}{\sqrt{d-2}} \Lambda^{\frac{1}{2}}\left\|\nabla u_{R, \delta}^{-}\right\|-\frac{\sqrt{2}}{\sqrt{d-2}} \epsilon(R)\right) .
\end{aligned}
$$

For fixed $R$, let $\delta \rightarrow 0$ in the last inequality; since $u_{R, \delta}$ is compactly supported, by the dominated convergence theorem, one gets

$$
\begin{aligned}
& \left(1-\frac{2(2 d-3)}{d-2} \Lambda-\frac{\sqrt{2}}{\sqrt{d-2}} \Lambda^{\frac{3}{2}}\right) \int\left|\nabla u_{R}^{-}\right|^{2}+\frac{\left|\lambda_{2}\right|}{\lambda_{1}^{\frac{1}{2}}} \frac{d-3}{d-1} \int|x|\left|\nabla u_{R}^{-}\right|^{2} \\
& \leq \epsilon^{2}(R)\left\|\nabla u_{R}^{-}\right\|\left(\frac{4 d-6}{d-2}+\frac{\sqrt{2}}{\sqrt{d-2}} \Lambda^{\frac{1}{2}}\left\|\nabla u_{R}^{-}\right\|-\frac{\sqrt{2}}{\sqrt{d-2}} \epsilon(R)\right) .
\end{aligned}
$$

Let finally $R \rightarrow \infty$; by the monotone convergence theorem and the fact that $u^{-} \in H^{1}\left(\mathbb{R}^{d}\right)$, we conclude that

$$
\left(1-\frac{2(2 d-3)}{d-2} \Lambda-\frac{\sqrt{2}}{\sqrt{d-2}} \Lambda^{\frac{3}{2}}\right) \int\left|\nabla u^{-}\right|^{2}+\frac{\left|\lambda_{2}\right|}{\lambda_{1}^{\frac{1}{2}}} \frac{d-3}{d-1} \int|x|\left|\nabla u^{-}\right|^{2} \leq 0 .
$$

By virtue of (50), it follows that $u^{-}$and thus $u$ are identically equal to zero.

Case $\left|\lambda_{2}\right|>\lambda_{1}$. Let $u \in H^{1}\left(\mathbb{R}^{d}\right)$ be a solution to (47). Choosing as a test function $v= \pm u$ in (47), and taking real and imaginary parts of the resulting identities, one easily gets

$$
\left(\lambda_{1} \pm \lambda_{2}\right) \int|u|^{2}=\int|\nabla u|^{2}+\Re \int f \bar{u} \pm \Im \int f \bar{u} .
$$

By the Schwarz inequality, the Hardy inequality (6) and assumption (49), we estimate

$$
\Re \int f \bar{u} \pm \Im \int f \bar{u} \leq 2 \int|f||u| \leq 2\|x f\|\left\|\frac{u}{|x|}\right\| \leq \frac{4}{d-2} \Lambda \int|\nabla u|^{2} .
$$

Consequently, (69) yields

$$
\left(\lambda_{1} \pm \lambda_{2}\right) \int|u|^{2} \geq\left(1-\frac{4}{d-2} \Lambda\right) \int|\nabla u|^{2} .
$$

Notice that (50) implies that $\Lambda<\frac{d-2}{4}$, therefore the last inequality forces $\lambda_{1} \pm \lambda_{2} \geq 0$ unless $u=0$. Since we assume $\left|\lambda_{2}\right|>\lambda_{1}$, we conclude that $u=0$. 
By taking $f:=V u$ in Theorem 8 (notice that $V u$ belongs to $H^{-1}\left(\mathbb{R}^{d}\right)$ under the hypothesis (70)) and using that $|u|=\left|u^{-}\right|$, we immediately obtain

Corollary 1. Let $d \geq 3$ and suppose

$$
\forall \psi \in H^{1}\left(\mathbb{R}^{d}\right), \quad \int_{\mathbb{R}^{d}}|x|^{2}|V(x)|^{2}|\psi(x)|^{2} d x \leq \Lambda^{2} \int_{\mathbb{R}^{d}}|\nabla \psi|^{2},
$$

where $\Lambda$ satisfies (50). Then $\sigma_{\mathrm{p}}\left(H_{V}\right)=\varnothing$.

Proof. In fact, Theorem 8 only gives the weaker conclusion that no complex point $\lambda$ satisfying $\Re \lambda>0$ can be an eigenvalue of $H_{V}$. However, (70) with (50) implies (1), which in turn yields that all possible eigenvalues of $H_{V}$ are included in the right complex plane, i.e. $\Re \lambda>0$. Indeed, this fact follows from the identity

$$
\int|\nabla u|^{2}+\Re \int V|u|^{2}=\Re \lambda \int|u|^{2},
$$

which can be obtained from (56) with the constant choice $G_{1}:=1$ and $f:=V u$.

Now we are in a position to prove Theorem 2

Proof of Theorem 2. Theorem 2 follows as a weaker version of Corollary 1 Indeed, it is easy to see that any $\Lambda$ verifying (50) necessarily satisfies $\Lambda \leq(d-2) / 2$. Using the latter in the former, we obtain (10) as a sufficient condition which guarantees (70).

We now turn our attention to Theorem 3 In analogy with the above strategy, we first study the (more difficult) part $\Re \lambda>0$. In the following, we set $V_{1}:=\Re V$ and $V_{2}:=\Im V$.

Theorem 9. Let $d \geq 3$. Let $u \in \mathcal{D}\left(\mathbb{R}^{d}\right)$ be a solution of (46) with $\Re \lambda>0$, and let $f:=V u$ where $V$ satisfies (14), (15), (16) and (13). Then $u=0$.

Proof. The proof is completely analogous to that of Theorem 8 . The only difference consists in the way we handle the right-hand side of (62), as we see in the sequel.

Case $\left|\lambda_{2}\right| \leq \lambda_{1}$. With the same notations as above, if $u \in \mathcal{D}\left(\mathbb{R}^{d}\right) \subset H^{1}\left(\mathbb{R}^{d}\right)$ solves (46)), then identity (62) holds. We now need to rewrite the right-hand side of (62) in a suitable way. To this aim, recall that $\widetilde{f}_{R}$ is defined via (52), where $f:=V u$. It is convenient to introduce the notation

$$
K_{R}(u, \nabla u):=-2 \nabla \xi_{R} \cdot \nabla u-u \Delta \xi_{R}
$$

so that $\tilde{f}_{R}=f_{R}+K_{R}(u, \nabla u)$. Putting (72) into (62), integrating by parts in the first two terms involving $V_{1}$ and taking the limit as $\delta \rightarrow 0$, one gets the following key identity:

$$
\begin{aligned}
& I:=\int\left|\nabla u_{R}^{-}\right|^{2}+\frac{\left|\lambda_{2}\right|}{\lambda_{1}^{\frac{1}{2}}} \int|x|\left|\nabla u_{R}^{-}\right|^{2}-\frac{(d-1)}{2} \frac{\left|\lambda_{2}\right|}{\lambda_{1}^{\frac{1}{2}}} \int \frac{\left|u_{R}\right|^{2}}{|x|}+\frac{\lambda_{2}}{\lambda_{1}^{\frac{1}{2}}} \int|x| V_{1}\left|u_{R}\right|^{2} \\
& =\underbrace{\int\left|u_{R}^{-}\right|^{2}\left(V_{1}+|x| \partial_{r} V_{1}\right)}_{I_{1}}+\underbrace{2 \Im \int|x| V_{2} u_{R}\left(\partial_{r} \overline{u_{R}}+i \operatorname{sgn}\left(\lambda_{2}\right) \lambda_{1}^{\frac{1}{2}} \overline{u_{R}}\right)}_{I_{2}} \\
& +\underbrace{(1-d) \Re \int K_{R}(u, \nabla u) \overline{u_{R}}-2 \Re \int|x| K_{R}(u, \nabla u)\left(\partial_{r} \overline{u_{R}}+i \operatorname{sgn}\left(\lambda_{2}\right) \lambda_{1}^{\frac{1}{2}} \overline{u_{R}}\right)-\frac{\lambda_{2}}{\lambda_{1}^{\frac{1}{2}} \Re \int|x| K_{R}(u, \nabla u) \overline{u_{R}}}}_{I_{3}} .
\end{aligned}
$$

We start by estimating the individual terms on the right hand side of (73). Thanks to assumption (15), we have

$$
I_{1}=\int\left|u_{R}^{-}\right|^{2} \partial_{r}\left(|x| V_{1}\right) \leq \int\left|u_{R}^{-}\right|^{2}\left[\partial_{r}\left(|x| V_{1}\right)\right]_{+} \leq b_{2}^{2} \int\left|\nabla u_{R}^{-}\right|^{2} .
$$


We now use $\partial_{r} \bar{u}_{R}+i \lambda_{1}^{\frac{1}{2}} \operatorname{sgn}\left(\lambda_{2}\right) \overline{u_{R}}=\overline{\partial_{r} u_{R}^{-}}$to write

$$
\left|I_{2}\right| \leq 2\left\|x V_{2} u_{R}\right\|\left\|\partial_{r} u_{R}^{-}\right\| \leq 2\left\|x V_{2} u_{R}\right\|\left\|\nabla u_{R}^{-}\right\| \leq 2 b_{3} \int\left|\nabla u_{R}^{-}\right|^{2} .
$$

Finally, by (51) and the fact that $u_{R} \in H^{1}\left(\mathbb{R}^{d}\right)$, one easily gets that

$$
\left|I_{3}\right| \leq \epsilon^{2}(R), \quad \lim _{R \rightarrow \infty} \epsilon^{2}(R)=0 .
$$

We now proceed by estimating the left-hand side of (73) from below. By (14) we obtain

$$
\frac{\left|\lambda_{2}\right|}{\lambda_{1}^{\frac{1}{2}}} \int|x| V_{1}\left|u_{R}\right|^{2} \geq-\left.\left.\frac{\left|\lambda_{2}\right|}{\lambda_{1}^{\frac{1}{2}}} \int\left(V_{1}\right)_{-}|| x\right|^{\frac{1}{2}} u_{R}^{-}\right|^{2} \geq-b_{1}^{2} \frac{\left|\lambda_{2}\right|}{\lambda_{1}^{\frac{1}{2}}} \int\left|\nabla\left(|x|^{\frac{1}{2}} u_{R}^{-}\right)\right|^{2} .
$$

Now write

$$
\frac{\left|\lambda_{2}\right|}{\lambda_{1}^{\frac{1}{2}}} \int|x|\left|\nabla u_{R}^{-}\right|^{2}-\frac{(d-1)}{2} \frac{\left|\lambda_{2}\right|}{\lambda_{1}^{\frac{1}{2}}} \int \frac{\left|u_{R}\right|^{2}}{|x|}=\frac{\left|\lambda_{2}\right|}{\lambda_{1}^{\frac{1}{2}}} \int\left|\nabla\left(|x|^{\frac{1}{2}} u_{R}^{-}\right)\right|^{2}-\frac{1}{4} \frac{\left|\lambda_{2}\right|}{\lambda_{1}^{\frac{1}{2}}} \int \frac{\left|u_{R}^{-}\right|^{2}}{|x|} .
$$

Notice that identity (57) with the constant choice $G_{2}(x):=\frac{\lambda_{2}}{\lambda_{2}}$, in the limit as $\delta \rightarrow 0$, reads as follows

$$
\left|\lambda_{2}\right| \int\left|u_{R}\right|^{2}=\frac{\lambda_{2}}{\left|\lambda_{2}\right|} \int V_{2}\left|u_{R}\right|^{2}+\frac{\lambda_{2}}{\left|\lambda_{2}\right|} \Im \int K_{R}(u, \nabla u) \overline{u_{R}} .
$$

Since $u_{R} \in H^{1}\left(\mathbb{R}^{d}\right)$, arguing as in (11), by (16), (51) and the fact that $\left|u_{R}\right|=\left|u_{R}^{-}\right|$, we obtain the $L^{2}$-bound

$$
\left\|u_{R}\right\|^{2} \leq\left|\lambda_{2}\right|^{-1}\left(\frac{2 b_{3}}{d-2} \int\left|\nabla u_{R}^{-}\right|^{2}+\epsilon^{2}(R)\right), \quad \lim _{R \rightarrow \infty} \epsilon^{2}(R)=0 .
$$

As a consequence of (79), since $\left|\lambda_{2}\right| \leq \lambda_{1}$, we can estimate the last term in (78), by the Schwarz and Hardy inequalities as follows:

$$
\frac{\left|\lambda_{2}\right|}{\lambda_{1}^{\frac{1}{2}}} \int \frac{\left|u_{R}^{-}\right|^{2}}{|x|} \leq \frac{\left|\lambda_{2}\right|}{\lambda_{1}^{\frac{1}{2}}}\left\|\frac{u_{R}^{-}}{|x|}\right\|\left\|u_{R}^{-}\right\| \leq \sqrt{b_{3}}\left(\frac{2}{d-2}\right)^{\frac{3}{2}} \int\left|\nabla u_{R}^{-}\right|^{2}+\frac{2}{d-2}\left\|\nabla u_{R}^{-}\right\||\epsilon(R)|,
$$

where $\epsilon(R)$ is the error term from (79). By (77), (78), and (80), we conclude that

$$
I \geq\left[1-\frac{1}{4} \sqrt{b_{3}}\left(\frac{2}{d-2}\right)^{\frac{3}{2}}\right] \int\left|\nabla u_{R}^{-}\right|^{2}-\frac{1}{4} \frac{2}{d-2}\left|\nabla u_{R}^{-} \|\right| \epsilon(R) \mid .
$$

Applying (74), (75), (76) and (81) in (73), we obtain

$$
\left[1-b_{2}^{2}-2 b_{3}-\frac{1}{4} \sqrt{b_{3}}\left(\frac{2}{d-2}\right)^{\frac{3}{2}}\right] \int\left|\nabla u_{R}^{-}\right|^{2} \leq \epsilon^{2}(R)+\frac{1}{4} \frac{2}{d-2}\left\|\nabla u_{R}^{-}\right\||\epsilon(R)|,
$$

for any $R>0$, with $\lim _{R \rightarrow \infty} \epsilon^{2}(R)=0$. In the limit as $R \rightarrow \infty$, by the monotone convergence theorem, we finally get

$$
\left[1-b_{2}^{2}-2 b_{3}-\frac{1}{4} \sqrt{b_{3}}\left(\frac{2}{d-2}\right)^{\frac{3}{2}}\right] \int\left|\nabla u^{-}\right|^{2} \leq 0 .
$$

By virtue of (13), it follows that $u^{-}$and thus $u$ are identically equal to zero. 
Case $\left|\lambda_{2}\right|>\lambda_{1}$. The proofs in this case is based on identity (69). When $f:=V u$, it reads as follows:

$$
\left(\lambda_{1} \pm \lambda_{2}\right) \int|u|^{2}=\int|\nabla u|^{2}+\int V_{1}|u|^{2} \pm \int V_{2}|u|^{2} \geq \int|\nabla u|^{2}-\int\left(V_{1}\right)_{-}|u|^{2}-\left.\left|\int V_{2}\right| u\right|^{2} \mid .
$$

By means of (11), (14) and (16), we have

$$
\left(\lambda_{1} \pm \lambda_{2}\right) \int|u|^{2} \geq\left[1-b_{1}^{2}-\frac{2 b_{3}}{d-2}\right] \int|\nabla u|^{2} .
$$

Therefore, condition (13) implies that $\lambda_{1} \pm \lambda_{2} \geq 0$, and since $\left|\lambda_{2}\right|>\lambda_{1}$ we conclude that $u$ is identically zero.

Now we are in a position to prove Theorem 3 .

Proof of Theorem 3. Theorem 9] implies that $\sigma_{\mathrm{p}}\left(H_{V}\right) \cap\left\{\lambda_{1}>0\right\}=\varnothing$. In addition, if $\lambda_{1} \leq 0$, then choosing $v:=u$ in (47) and taking the resulting real part, one obtains

$$
\lambda_{1} \int|u|^{2}=\int|\nabla u|^{2}+\int V_{1}|u|^{2} \geq \int|\nabla u|^{2}-\int\left(V_{1}\right)_{-}|u|^{2} \geq\left(1-b_{1}^{2}\right) \int|\nabla u|^{2},
$$

where the last inequality follows by (14). This implies that $\sigma_{\mathrm{p}}\left(H_{V}\right) \cap\left\{\lambda_{1} \leq 0\right\}=\varnothing$, so the proof is completed.

We conclude the manuscript with the proof of Theorem 4. Since the strategy is identical to the proof of Theorem 3, we just sketch it.

Proof of Theorem 4. Equation (46) is now replaced by

$$
\Delta_{A} u+\lambda u=V u,
$$

where $\Delta_{A}:=\nabla_{A} \cdot \nabla_{A}$. Let $u \in \mathcal{D}_{A}\left(\mathbb{R}^{d}\right)$ be a weak solution to (184). By similar algebraic manipulations as in the proof of Theorem 3, we get an analogue to (73):

$$
\begin{aligned}
& \int\left|\nabla_{A} u_{R}^{-}\right|^{2}+\frac{\left|\lambda_{2}\right|}{\lambda_{1}^{\frac{1}{2}}} \frac{d-3}{d-1} \int|x|\left|\nabla_{A} u_{R}^{-}\right|^{2} \leq \int\left|u_{R}\right|^{2}\left(V_{1}+|x| \partial_{r} V_{1}\right)+\frac{\lambda_{2}}{\lambda_{1}^{\frac{1}{2}}} \int|x| V_{1}\left|u_{R}\right|^{2} \\
& +2 \Im \int|x| u_{R} V_{2}\left(\overline{\partial_{r}^{A} u_{R}}+i \operatorname{sgn}\left(\lambda_{2}\right) \lambda_{1}^{\frac{1}{2}} \overline{u_{R}}\right)-2 \Im \int|x| u_{R} B_{\tau} \cdot \overline{\nabla_{A} u_{R}} \\
& +(1-d) \Re \int K_{R}\left(u, \nabla_{A} u\right) \overline{u_{R}}-2 \Re \int|x| K_{R}\left(u, \nabla_{A} u\right)\left(\partial_{r}^{A} \overline{u_{R}}+i \operatorname{sgn}\left(\lambda_{2}\right) \lambda_{1}^{\frac{1}{2}} \overline{u_{R}}\right) \\
& -\frac{\lambda_{2}}{\lambda_{1}^{\frac{1}{2}}} \iint|x| K_{R}\left(u, \nabla_{A} u\right) \overline{u_{R}},
\end{aligned}
$$

where $\partial_{r}^{A}:=\frac{x}{|x|} \cdot \nabla_{A}$. In fact, in order to obtain the last identity, one proceeds exactly as above, with the only difference arising once obtaining identity (58), in which we use the test function $v:=$ $\nabla G_{3} \cdot \nabla_{A} u_{R, \delta}+\Delta G_{3} u_{R, \delta}$. The key remark is that $B_{\tau}$ is a tangential vector, so that

$$
B_{\tau} \cdot \nabla_{A} u=B_{\tau} \cdot\left(\nabla_{A} u+i \operatorname{sgn}\left(\lambda_{2}\right) \lambda_{1}^{\frac{1}{2}} \frac{x}{|x|} u\right)=B_{\tau} \cdot \nabla_{A} u_{R}^{-}
$$

and we can rewrite the last inequality as

$$
\begin{aligned}
& \int\left|\nabla_{A} u_{R}^{-}\right|^{2}+\frac{\left|\lambda_{2}\right|}{\lambda_{1}^{\frac{1}{2}}} \frac{d-3}{d-1} \int|x|\left|\nabla_{A} u_{R}^{-}\right|^{2} \\
& \leq \int\left|u_{R}\right|^{2}\left(V_{1}+|x| \partial_{r} V_{1}\right)+\frac{\lambda_{2}}{\lambda_{1}^{\frac{1}{2}}} \int|x| V_{1}\left|u_{R}\right|^{2}+2 \Im \int|x| u_{R} \overline{\nabla_{A} u_{R}} \cdot\left(V_{2} \frac{x}{|x|}-B_{\tau}\right) \\
& \quad+(1-d) \Re \int K_{R}\left(u, \nabla_{A} u\right) \overline{u_{R}}-2 \Re \int|x| K_{R}\left(u, \nabla_{A} u\right)\left(\partial_{r}^{A} \overline{u_{R}}+i \operatorname{sgn}\left(\lambda_{2}\right) \lambda_{1}^{\frac{1}{2}} \overline{u_{R}}\right) \\
& \quad-\frac{\lambda_{2}}{\lambda_{1}^{\frac{1}{2}}} \Re \int|x| K_{R}\left(u, \nabla_{A} u\right) \overline{u_{R}} .
\end{aligned}
$$


One now proceeds in the same way as in the magnetic-free case, to conclude that $u=0$ if $\Re \lambda>0$. To complete the proof, we then argue exactly as above; we omit further details.

\section{Acknowledgment}

The first two authors acknowledge the hospitality of the Basque Center for Applied Mathematics in Bilbao where this work was initiated and completed. The first author also acknowledges the hospitality of the Nuclear Physics Institute in Řž where this work was developed in part. The research was partially supported by the project RVO61389005, the GACR grant No. 14-06818S, and the MIUR grant FIRB 2012 - RBFR12MXPO-002.

\section{References}

[1] A. A. Abramov, A. Aslanyan, and E. B. Davies, Bounds on complex eigenvalues and resonances, J. Phys. A: Math. Gen. 34 (2001), 57-72.

[2] J. A. Barceló, L. Vega, and M. Zubeldia, The forward problem for the electromagnetic Helmholtz equation with critical singularities, Adv. Math. 240 (2013), 636-671.

[3] D. Borisov and D. Krejčiřík, PT-symmetric waveguides, Integ. Equ. Oper. Theory 62 (2008), 489515 .

[4] V. Bruneau and E. M. Ouhabaz, Lieb-Thirring estimates for non self-adjoint Schrödinger operators, J. Math. Phys. 49 (2008), 093504.

[5] N. Burq, F. Planchon, J. Stalker, and S. Tahvildar-Zadeh, Strichartz estimates for the wave and Schrödinger equations with potentials of critical decay, Indiana Univ. Math. J. 53 (2004), 1665-1680.

[6] M. Demuth, M. Hansmann, and G. Katriel, On the discrete spectrum of non-selfadjoint operators, J. Funct. Anal. 257 (2009), 2742-2759.

[7] _ Lieb-Thirring type inequalities for Schrödinger operators with a complex-valued potential, Integ. Equ. Oper. Theory 75 (2013), 1-5.

[8] D. E. Edmunds and W. D. Evans, Spectral theory and differential operators, Oxford University Press, Oxford, 1987.

[9] A. Enblom, Estimates for eigenvalues of Schrödinger operators with complex-valued potentials, Lett. Math. Phys., to appear; preprint on arXiv:1503.06337 [math.SP] (2015).

[10] L. Fanelli and L. Vega, Magnetic virial identities, weak dispersion and Strichartz estimates, Math. Ann. 344 (2009), 249-278.

[11] R. L. Frank, Eigenvalue bounds for Schrödinger operators with complex potentials, Bull. Lond. Math. Soc. 43 (2011), 745-750.

[12] R. L. Frank, A. Laptev, E. H. Lieb, and R. Seiringer, Lieb-Thirring inequalities for Schrödinger operators with complex-valued potentials, Lett. Math. Phys. 77 (2006), 309-316.

[13] R. L. Frank and B. Simon, Eigenvalue bounds for Schrödinger operators with complex potentials. II, J. Spectr. Theory, to appear; preprint on arXiv:1504.01144 [math.SP] (2015).

[14] T. Ikebe and Y. Saito, Limiting absorption method and absolute continuity for the Schrödinger operator, J. Math. Kyoto Univ. 12 (1972), 513-542.

[15] A. D. Ionescu and D. Jerison, On the absence of positive eigenvalues of Schrödinger operators with rough potentials, Geom. Funct. Anal. 13 (2003), 1029-1081.

[16] T. Kato, Perturbation theory for linear operators, Springer-Verlag, Berlin, 1966. 
[17] H. Koch and D. Tataru, Carleman estimates and absence of embedded eigenvalues, Commun. Math. Phys. 267 (2006), 419-449.

[18] D. Krejčirík and Z. Lu, Location of the essential spectrum in curved quantum layers, J. Math. Phys. 55 (2014), 083520.

[19] A. Laptev and O. Safronov, Eigenvalue estimates for Schrödinger operators with complex potentials, Comm. Math. Phys. 292 (2009), 29-54.

[20] M. Reed and B. Simon, Methods of modern mathematical physics, IV. Analysis of operators, Academic Press, New York, 1978.

[21] O. Safronov, Estimates for eigenvalues of the Schrödinger operator with a complex potential, Bull. Lond. Math. Soc. 42 (2010), 452-456.

[22] B. Simon, Quantum mechanics for Hamiltonians defined by quadratic forms, Princeton Univ. Press., New Jersey, 1971. 